\title{
Cell Surface Display System for Lactococcus Lactis: A Novel Development for Oral Vaccine
}

\begin{abstract}
The food - grade Lactococcus lactis is a potential vector to be used as a live vehichle for the delivery of heterologous proteins for vaccine and pharmaceutical purposes. We constructed a plasmid vector pSVac that harbors a 255 -bp single - repeat sequence of the cell wall binding protein region of the AcmA protein. The recombinant plasmid was transformed into Eschericia coli and expression of the gene fragment was driven by the T7 promoter of the plasmid. SDS-PAGE showed the presence of the putative AcmA ' fragment and this was confirmed by West-tern blot analysis. The protein was isolated and purified using a His - tag affinity column. When mixed with a culture of L. lactis MG 1363, ELISA and immunofluorescence assays showed that the cell wall - binding fragment was anchored onto the outer surface of the bacteria. This indicated that the AcmA' repeat unit retained the actice site for binding onto the cell wall surface of the L. lactis cells. Stability assays showed that the fusion proteins (AcmA/A1, AcmA/A3) were stably docked onto the surface for at least 5 days. The AcmA' fragment was also shown to be able to strongly bind onto the cell surface of naturally occurring lactococcal strains and Lactobacillus and, with less strength, the cell surface of Bacillus sphericus. The new system designed for cell surface display of recombinant proteins on L.lactis was evaluated for the expression and display of A1 and A3 regions of the VP1 protein of entero-virus 7I (EV7I). The A1 and A3 regions of the VP1 protein of EV7I were cloned upstream to the cell wall - binding domains of AcmA protein and successfully expressed as AcmA/A1 and AcmA/A3. Whole - cell ELISA showed the successful display of VP1 onto the surface of L. lactis. The success of the anchoring system developed in this study for docking the A1 and A3 epitopes of VP1 onto the surface of L lactis cell opens up the possibilities of peptide and protein display for not only Lactococcus but also for other gram - positive bacteria. This novel way of displaying epitopes on the cell surface of L. laptis and other related organisms should be very useful in the delivery of vaccines and other useful proteins.
\end{abstract}

Keyword: Chemistry and Materials Science 